\title{
A preliminary study of bilateral color mapping of pig bladder vasculature demonstrates potential for acute hemi-ischemic events
}

\author{
Zachary E. Cullingsworth ${ }^{1}$, Naveen Nandanan ${ }^{2}$, Natalie R. Swavely ${ }^{2}$, Konstantin Frolov ${ }^{1}$, Randy Vince ${ }^{2}$, \\ Rebecca Zee ${ }^{2}$, Theodore Cisu ${ }^{2}$, Adam P. Klausner ${ }^{2}$, John E. Speich ${ }^{1}$ \\ ${ }^{1}$ Department of Mechanical and Nuclear Engineering, Virginia Commonwealth University School of Engineering, Richmond, Virginia, USA; \\ ${ }^{2}$ Department of Surgery/Division of Urology, Virginia Commonwealth University School of Medicine, Richmond, Virginia, USA \\ Contributions: (I) Conception and design: ZE Cullingsworth, K Frolov, R Vince, AP Klausner, JE Speich; (II) Administrative support: AP Klausner, \\ JE Speich; (III) Provision of study materials/patients: N Nandanan, NR Swavely, R Vince; (IV) Collection and assembly of data: ZE Cullingsworth, \\ N Nandanan, K Frolov; (V) Data analysis and interpretation: ZE Cullingsworth, T Cisu, R Zee, AP Klausner, JE Speich, K Frolov; (VI) Manuscript \\ writing: All authors; (VII) Final approval of manuscript: All authors. \\ Correspondence to: John E. Speich, PhD. Department of Mechanical and Nuclear Engineering, Virginia Commonwealth University, 401 West Main \\ Street, PO Box 843015, Richmond, 23284, Virginia, USA. Email: jespeich@vcu.edu.
}

Background: Chronic ischemia is a known risk factor for the development of lower urinary tract symptoms (LUTS) and bladder hypocontractility. Less is known, however, about the impact of acute ischemia. Classic teaching suggests that collateral circulation is robust in the bladder and, therefore, loss of a single source of blood flow should have no deleterious effect. This study aims to provide visual evidence that segmental vascular supply is critical for maintaining adequate perfusion to the bladder.

Methods: Ex vivo pig bladders were cannulated bilaterally in the superior vesical arteries and perfused using contrasting red and green dye. Images were collected at each step of the perfusion dyeing process and these images were analyzed using a custom program to calculate the average hue of each side. Statistical analysis was performed using Student's $t$-test.

Results: The two halves being perfused by separate arteries showed a statistically significant difference when compared $(\mathrm{P}<0.05)$ on both the outer wall $(\mathrm{n}=9)$ and in the mucosal layer $(\mathrm{n}=4)$. On the outside wall, the average normalized hue of the green halves was $27.5^{\circ} \pm 14.3^{\circ}$, while the average normalized hue of the red halves was $-58.7^{\circ} \pm 3.1^{\circ}$. In the mucosa, the average normalized hue of the green halves was $34.5^{\circ} \pm 17.4^{\circ}$, while the average normalized hue of the red halves was $-51.5^{\circ} \pm 3.5^{\circ}$.

Conclusions: This study identified a novel color mapping method to study pig bladder vascular supply. The results demonstrated a lack of collateral blood flow, highlighting the possibly of acute hemi-ischemic event. However, further research in the effect of acute ischemia on bladder function is necessary.

Keywords: Urinary bladder; ischemia; transient ischemic attack

Submitted Nov 30, 2020. Accepted for publication Apr 16, 2021.

doi: $10.21037 /$ tau-20-1467

View this article at: http://dx.doi.org/10.21037/tau-20-1467

\section{Introduction}

Lower urinary tract symptoms (LUTS) represent a bothersome constellation of symptoms that are common in the urologic patient population. Aging has been implicated as an independent risk factor for development of LUTS in both men and women. Vascular diseases common to aging, such as atherosclerosis, may play a role in the development of chronic bladder ischemia (1). It has been demonstrated that LUTS increases with age, and these patients have decreased bladder blood flow compared 

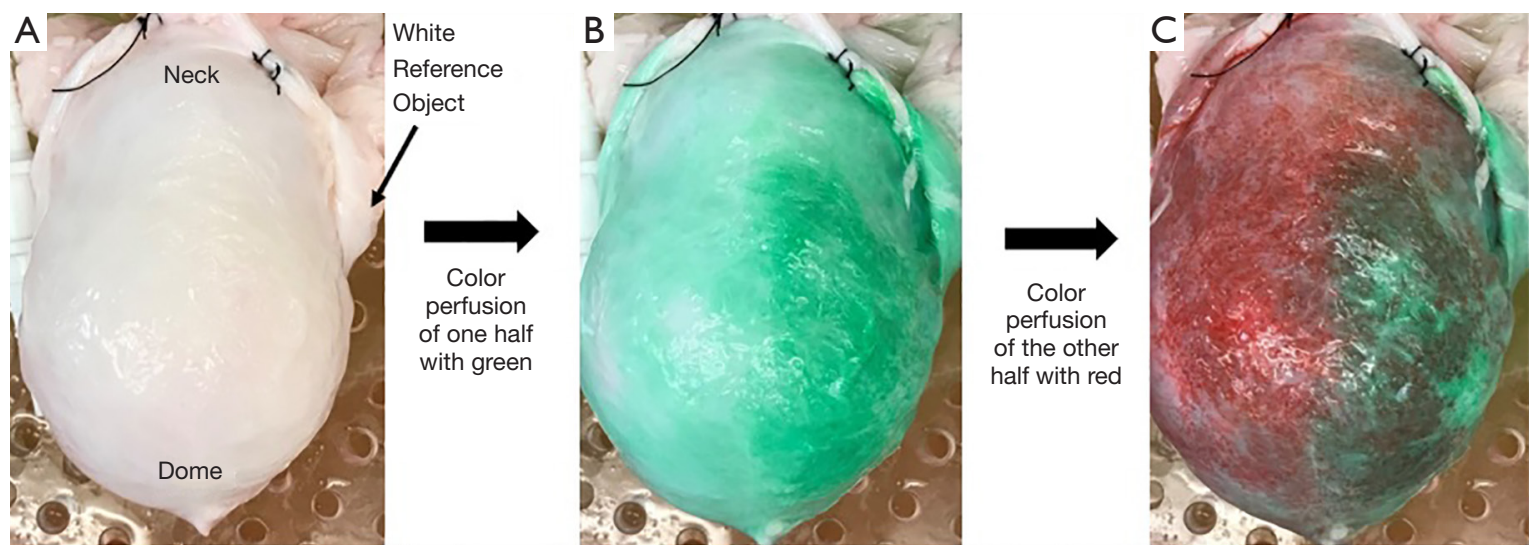

Figure 1 Bladder mapping in 3 stages: (A) before being dyed, (B) green dye injected into right vesical artery, (C) red dye injected into left vesical artery.

to younger asymptomatic patients (2-4). A suggested mechanism has been proposed in which prolonged ischemia leads to detrusor overactivity (DO), followed eventually by detrusor underactivity (5-8). Additionally, new mechanisms for treatment of LUTS, based on the etiology of pelvic ischemia, have been emerging and are a current area of active research (9-14).

Molecular mechanisms of ischemic insult causing deficits in bladder function are incompletely elucidated. Animal experiments have demonstrated that chronic bladder ischemia leads to detrusor denervation, upregulation of proinflammatory cytokines such as HIF$1 \mathrm{a}$, increased collagen deposition, and generation of reactive oxygen species (ROS) (15-20). Blood flow to the bladder is supplied by the paired superior and inferior vesical arteries. Classic teaching dictates that loss of one source of blood supply to the bladder would be inconsequential owing to robust collateral circulation. However, there is a paucity of literature to support this. Rabbit studies of acute unilateral bladder ischemia have demonstrated short term changes in gene expression similar to that seen in BOO, detrusor hyperreactivity and loss of compliance (21-23).

Deciphering the distribution of perfusion within the bladder may be important in underactive bladder pathophysiology that occurs in the initial stages of chronic ischemia. The aim of this study is to provide visual evidence that adequate bladder perfusion is maintained by a segmental vascular supply. We present the following article in accordance with the ARRIVE reporting checklist (available at http://dx.doi. org/10.21037/tau-20-1467).

\section{Methods}

\section{Bladder harvesting}

As previously described (24-28), bladders for this study were procured from local abattoirs immediately after slaughter. Experiments were performed and granted exempt status after review by the Virginia Commonwealth University institutional care and use committee because the animals were already deceased at time of tissue harvest. Briefly, the entire urinary tract was removed en bloc with intact vasculature to the aorta and vena cava. The vasculature was flushed with a heparinized Krebs Henseleit solution. The kidneys and any excess tissue were removed leaving the ureters, bladder, urethra and vasculature intact, which were then placed into a physiologic solution on ice for preservation during transport. In the lab, the superior vesical arteries were cannulated bilaterally via the aorta. The cannulas were then advanced through the internal iliac arteries and secured in place with 2-0 silk ties. Bilateral ureters were tied off with 2-0 silk ties.

\section{Filling and dying}

After cannulation, bladders were placed in a humidity chamber to simulate physiologic conditions. An infusion catheter was inserted in the urethra to fill the bladder, which was filled to $250 \mathrm{~mL}$ at a rate of $50 \mathrm{~mL} / \mathrm{min}$, allowing for sufficient size for image collection (Figure 1A). This volume was selected based on our previously published experiments and those of others (14,24-28). During dye infusion, the 


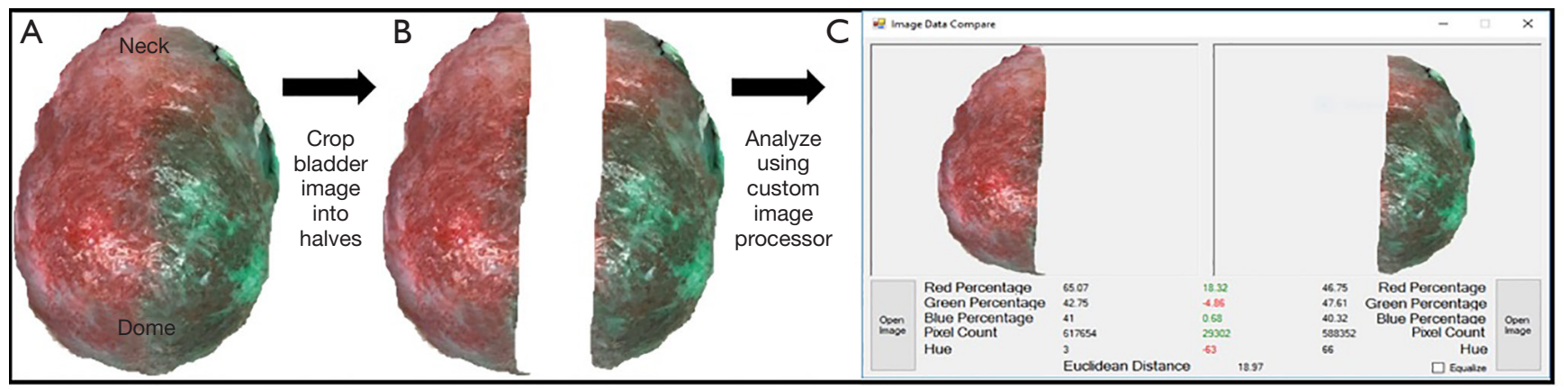

Figure 2 Bladder analysis steps. (A) Whole bladder, (B) separate images for each half, (C) images loaded into custom program for analysis.

cannulated arteries were perfused at a rate of $10 \mathrm{~mL} / \mathrm{min}$ to approximate a high physiologic flow rate (25). The first artery was perfused with deionized water mixed with standard green food coloring. Green was used first to show sharpest contrast against the naturally pink tinted bladder (Figure $1 \mathrm{~B}$ ). Once $50 \mathrm{~mL}$ had been perfused, deionized water mixed with red food coloring was perfused through the contralateral artery to provide a distinct color difference from the already dyed green region (Figure 1C).

\section{Image collection}

The bladder work was performed in a well-lit room with adequate lighting to minimize the noise in the resulting images. The photographs were taken using the standard Apple iPhone built-in camera. These were taken with JPEG compression (94\% quality) and with auto exposure and auto white balance. The camera was placed level on an arm located 0.45 meters above the bladder work area. Images were taken at various stages of the bladder dye protocol: before any color perfusion, after the green perfusion, and after the red perfusion. After initial images were collected, several bladders were also divided along the midline, demonstrating vasculature differences in the two hemi-bladders via the visualized differences in green and red coloration. Bladders were then opened and an additional image was collected, showing the dye-visualized vascular perfusion of the mucosal surface of the bladder. Each image included the same white color reference object, which was used to correct the white balance during processing.

\section{Image processing}

The images were first color corrected using the white object included in the original image for consistency. The background in the bladder image was then removed (Figure $2 A$ ) and the image was split into two halves following along the line between the dome and base of the bladder (Figure 2B). The two halves were exported as PNG files so as to not further compress the image, and to keep the transparency component of the image. These segmented images were inputted into a custom written program for analysis (Figure 2C). The mucosal images of the bladder were processed in a similar manner (Figure 3).

\section{Image analysis}

The custom program used to calculate the color values present in the image used popular C-Sharp (C\#) library, Accord.NET Framework, for color calculations. The program takes an image input and calculates the mean Red, Green, Blue (RGB) across the entire image using a color histogram calculated from that image. This mean RGB value is converted from standard Red, Green, Blue (sRGB) into the Hue Saturation Value (HSV) color space. The hue of the average color is recorded. Values are reported as mean \pm standard error and statistical analysis was done using paired $t$-tests with $\mathrm{P}<0.05$ indicating statistical significance.

\section{Hue, saturation, value}

The HSV color model is a method of describing color in a manner that is more related to how colors are perceived. Hue is the color segment of this model, and is calculated as a value between $0^{\circ}$ and $360^{\circ}$ with each degree corresponding to a color value, with $0^{\circ} / 360^{\circ}$ representing pure red, $120^{\circ}$ pure green and $240^{\circ}$ pure blue. Saturation refers to the amount of gray in a color. It represents the radius from the center on the HSV wheel and has a value between $0-1$, with 

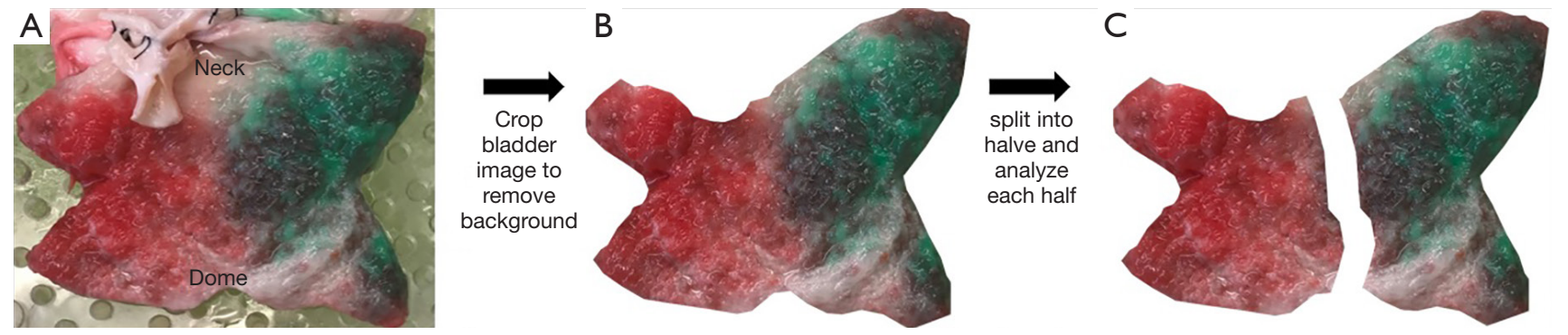

Figure 3 Internal bladder analysis steps. (A) Entire split bladder, (B) image separated from background, (C) images split and loaded into custom program for analysis.

0 being completely gray. Value describes the brightness of a color and varies from $0-100 \%$, with $0 \%$ being completely black. Only the hue is considered in the current analysis. The hue of each bladder half was normalized to $60^{\circ}$ for comparison, which corresponds to yellow, and is directly between red and green, the colors used for perfusion in this experiment.

\section{Results}

A total of nine bladders were utilized for outer surface image analysis (Figure 4) and four of these bladders were divided for additional mucosal surface image analysis (Figure $3 A$ ). After processing and analysis, two distinct halves were found for each color corresponding to unilateral cannulated arteries. On the outer surface of the bladder $(n=9)$, there was a statistically significant difference in the color hue between the two hemi-bladders. The average normalized hue of the green halves was $27.5^{\circ} \pm 14.3^{\circ}$, corresponding to $87.5^{\circ}$ with pure green being $120^{\circ}$, while the average of the red halves was $-58.7^{\circ} \pm 3.1^{\circ}$, corresponding to $1.3^{\circ}$ with pure red being $0^{\circ}(\mathrm{P}=0.0003)$. The inner surface of the bladders $(\mathrm{n}=4)$ also showed a statistically significant difference $(\mathrm{p}=0.0088)$ between the hemi-bladders, with the average normalized hue of the green halves being $34.5^{\circ} \pm 17.4^{\circ}$, corresponding to $94.5^{\circ}$ and the average hue of the red halves was $-51.5^{\circ} \pm 3.5^{\circ}$, corresponding to $8.5^{\circ}$.

Hue refers to an angle on the HSV color wheel, which can aide in the visualization of the average color. The average hue of each bladder half is represented as a line drawn on a color wheel relative to the color yellow (Figure 5). On the outer bladder wall, the green hue of the green halves was significantly different than the hue of the red halves. Similarly, the inner bladder walls display a significant difference in hues when comparing the red and green halves (Figure 5).

\section{Discussion}

There is limited understanding of the vascular distribution that exists in the urinary bladder although urologists are widely taught that the bladder has excellent collateral circulation (29). Through our novel color mapping method with colored dye infusion into the paired superior vesical arteries, we demonstrate that there are areas of the bladder that are supplied chiefly by the ipsilateral artery. Each hemibladder demonstrated a statistically significantly different hue than the contralateral side suggesting reliance on a specific blood supply for perfusion. There is some degree of collateral circulation, as is noted in the hue analysis, that neither red nor green was a pure color.

Pelvic ischemia caused by atherosclerosis in aging is thought to play a role in the pathophysiology of LUTS, development of DO and subsequent progression to detrusor underactivity (5-8). Understanding the distribution of vascular flow is crucial to investigating the effect of ischemia on the urinary bladder and whether a hemi-ischemic event in the setting of transient or chronic ischemia is possible. The functional and molecular effect of acute ischemia has been investigated in a rabbit model (30). Rabbit detrusor smooth muscle displayed a significant decrease in contractile responses and compliance after bilateral ischemia $(22,23)$.

Correspondingly, unilateral ischemia has been tested in rabbit models. Unilateral ligation of vesical arteries for 1 week resulted in $74 \%$ and $32 \%$ reduction in contractile response to bethanechol on the ipsilateral and contralateral sides, respectively (22). There was a corresponding $35 \%$ reduction in bladder capacity after 1 week suggesting 


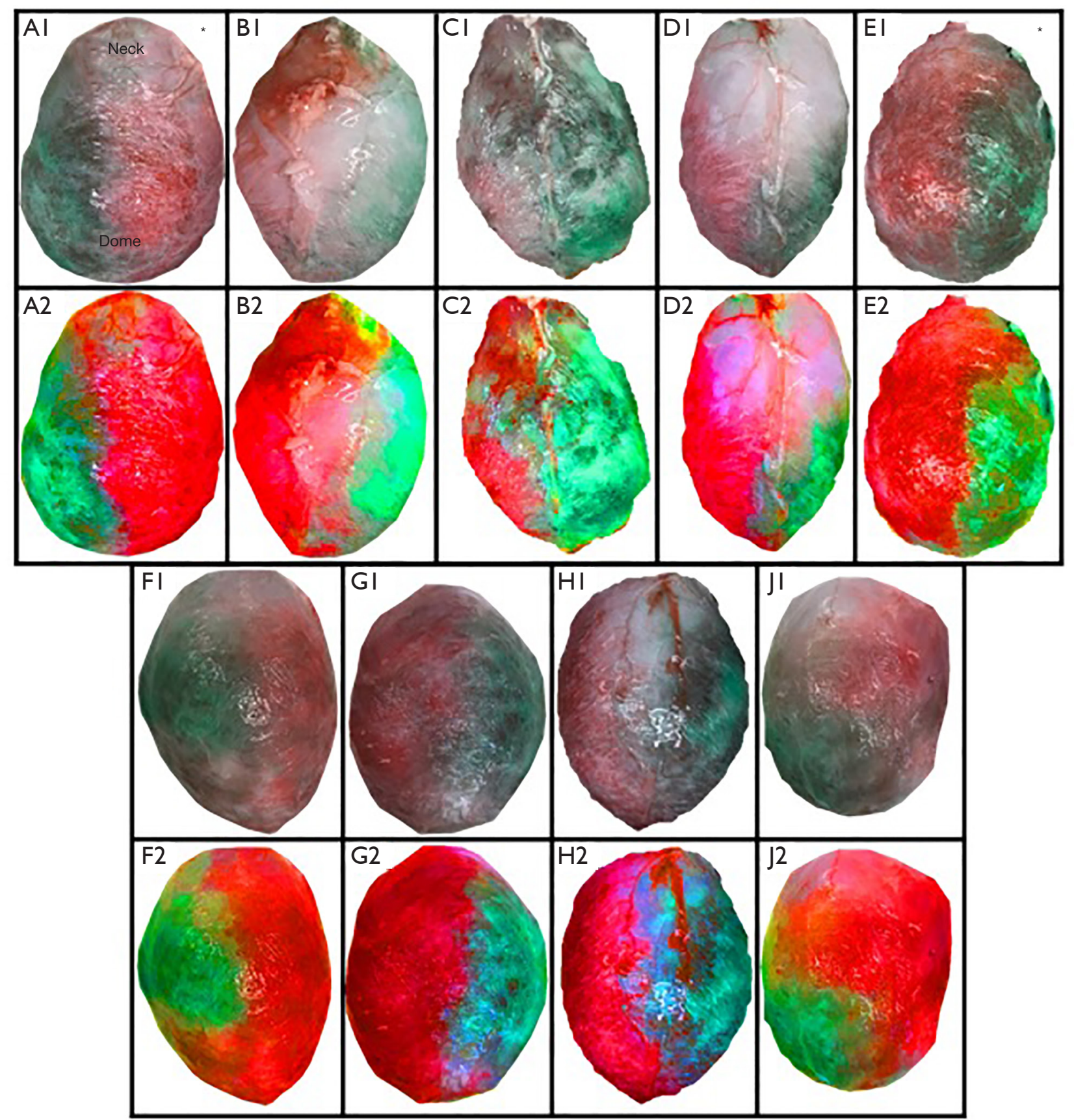

Figure 4 Images of all raw dyed bladders used for analysis (A1-J1), with corresponding bladders with exaggerated saturation to show the contrast shown below them (A2-J2) (*indicating female bladder).

functional consequences of unilateral ischemia (22). mRNA expression was altered in both the ischemic and nonischemic sides of the bladder, with increased expression of c-fos, c-jun, bFGF, and hsp-70 mRNA transcripts observed on the ischemic side (21). These alterations were expressed in a pattern similar to the changes seen in rabbit models of partial bladder outlet obstruction and bladder overdistension, both conditions known to cause 

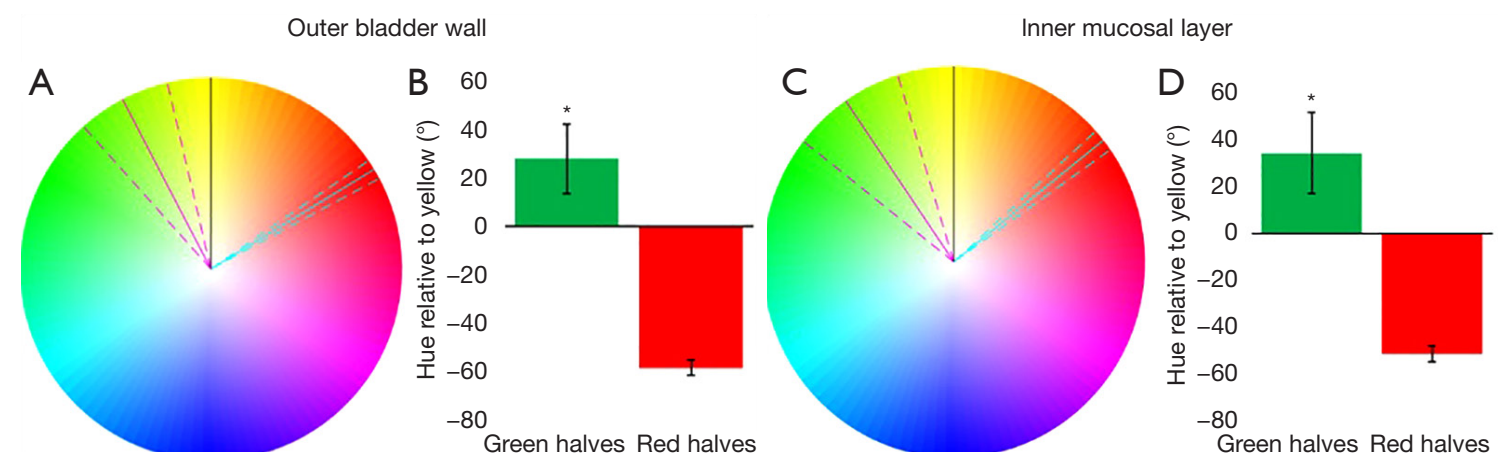

Figure 5 Comparison of hues on the inner and outer walls of the bladder. (A) HSV (hue, saturation, value) wheel with yellow $\left(60^{\circ}\right.$ hue) set as a vertical reference (black line), the average hue of the red (cyan lines \pm dashed lines) and the average hue of the green (magenta lines \pm dashed lines) compared to the reference. (B) Bar chart showing average values of bladder hue by halves in degrees ( $\mathrm{n}=9)$ using yellow $\left(60^{\circ}\right.$ hue) as a reference point (* indicating significant difference). (C) HSV wheel with yellow ( $60^{\circ}$ hue) set as a vertical reference (black line), the average hue of the red mucosal halves of the bladders (cyan lines \pm dashed lines) and the average hue of the green mucosal halves of the bladders (magenta lines \pm dashed lines) compared to the reference. (D) Bar chart showing average values of internal bladder hue by halves in degrees $(n=4)$ using yellow $\left(60^{\circ}\right.$ hue $)$ as a reference point (*indicating significant difference).

bladder ischemia (21). Taken, together, these data support our hypothesis that bladder blood flow has a geographic distribution and collateral circulation that may not be able to compensate for loss of part of the bladder's blood supply with implications for both gene expression as well as bladder function.

There are limitations to the current study. It is not known whether dyes used in this study diffuse through bladder at the same rate as blood, and certainly, the color wheel normalization used in this study may not account for issues related to dye mixing. In this initial colorimetric study, physiologic buffer was not used due to the acute nature of the imaging. Future studies aimed at the evaluation of organ function in the setting of ischemia should use physiologic solutions. Ex vivo blood flow may not be the same as in the in vivo states, and the pig bladder, while similar to humans, may not have identical vascular supply. Furthermore, the pressure used to force the dye into the bladder vasculature may have been supraphysiologic, potentially causing greater crossover to be observed in this study than would normally occur in vivo. Finally, the study did not measure tissue function or biochemical/molecular measures of ischemia. However, this study aimed mainly to identify a simple visual method to map bladder perfusion on both the inner and outer surfaces. To our knowledge, this is the first study demonstrating color perfusion of bladder tissue in order to characterize bladder vascular distribution. Further research is needed to investigate the effects of acute ischemia on bladder function.

\section{Conclusions}

Perfusion of each hemi-bladder with contrasting dyes demonstrates a lack of collateral vesical blood flow suggesting that there are regions in the bladder at risk for ischemia with a loss of blood supply. Further research is warranted to understand bladder perfusion as well as the effect of acute ischemia on bladder function.

\section{Acknowledgments}

Funding: None.

\section{Footnote}

Reporting Checklist: The authors have completed the ARRIVE reporting checklist. Available at http://dx.doi. org/10.21037/tau-20-1467

Data Sharing Statement: Available at http://dx.doi. org/10.21037/tau-20-1467

Peer Review File: Available at http://dx.doi.org/10.21037/ tau-20-1467

Conflicts of Interest: All authors have completed the ICMJE uniform disclosure form (available at http://dx.doi. org/10.21037/tau-20-1467). The authors have no conflicts of interest to declare. 
Ethical Statement: The authors are accountable for all aspects of the work in ensuring that questions related to the accuracy or integrity of any part of the work are appropriately investigated and resolved. Experiments were performed and granted exempt status after review by the Virginia Commonwealth University institutional care and use committee because the animals were already deceased at time of tissue harvest.

Open Access Statement: This is an Open Access article distributed in accordance with the Creative Commons Attribution-NonCommercial-NoDerivs 4.0 International License (CC BY-NC-ND 4.0), which permits the noncommercial replication and distribution of the article with the strict proviso that no changes or edits are made and the original work is properly cited (including links to both the formal publication through the relevant DOI and the license). See: https://creativecommons.org/licenses/by-nc-nd/4.0/.

\section{References}

1. Speich JE, Tarcan T, Hashitani H, et al. Are oxidative stress and ischemia significant causes of bladder damage leading to lower urinary tract dysfunction? Report from the ICI-RS 2019. Neurourol Urodyn 2020;39 Suppl 3:S16-22.

2. De EJ, Hou P, Estrera AL, et al. Pelvic ischemia is measurable and symptomatic in patients with coronary artery disease: a novel application of dynamic contrastenhanced magnetic resonance imaging. J Sex Med 2008;5:2635-45.

3. Pinggera GM, Mitterberger M, Pallwein L, et al. alphaBlockers improve chronic ischaemia of the lower urinary tract in patients with lower urinary tract symptoms. BJU Int 2008;101:319-24.

4. Pinggera G-M, Mitterberger $M$, Steiner E, et al. Association of lower urinary tract symptoms and chronic ischaemia of the lower urinary tract in elderly women and men: assessment using colour Doppler ultrasonography. BJU Int 2008;102:470-4.

5. Sagawa K, Aikawa K, Nomiya M, et al. Impaired detrusor contractility in a rat model of chronic bladder ischemia. Urology 2013;81:1379.e9-1379.e14.

6. Nomiya M, Yamaguchi O, Akaihata H, et al. Progressive vascular damage may lead to bladder underactivity in rats. J Urol 2014;191:1462-9.

7. Zhang Q, Siroky M, Yang JH, et al. Effects of ischemia and oxidative stress on bladder purinoceptors expression.
Urology 2014;84:1249.e1-1249.e7.

8. Zhao Z, Azad R, Yang JH, et al. Progressive changes in detrusor function and micturition patterns with chroinc bladder ischemia. Investig Clin Urol 2016;57:249-59.

9. Goi $Y$, Tomiyama $Y$, Nomiya $M$, et al. Effects of silodosin, a selective alpha1A-adrenoceptor antagonist, on bladder blood flow and bladder function in a rat model of atherosclerosis induced chronic bladder ischemia without bladder outlet obstruction. J Urol 2013;190:1116-22.

10. Sawada N, Nomiya M, Hood B, et al. Protective effect of a beta3-adrenoceptor agonist on bladder function in a rat model of chronic bladder ischemia. Eur Urol 2013;64:664-71.

11. Andersson KE, Nomiya M, Yamaguchi O. Chronic Pelvic Ischemia: Contribution to the Pathogenesis of Lower Urinary Tract Symptoms (LUTS): A New Target for Pharmacological Treatment? Low Urin Tract Symptoms 2015;7:1-8.

12. Goi Y, Tomiyama Y, Maruyama I, et al. Silodosin, an alpha(1A)-Adrenoceptor Antagonist, May Ameliorate Ischemia-Induced Bladder Denervation and Detrusor Dysfunction by Improving Bladder Blood Flow. Pharmacology 2016;97:161-70.

13. Shim JS, Bae JH. Drug Concentration in Rat Plasma, Bladder, and Prostate After Mirodenafil Administration in a Chronic Pelvic Ischemia Model. Urology 2016;91:244. e1-244.e5.

14. Andersson KE, Boedtkjer DB, Forman A. The link between vascular dysfunction, bladder ischemia, and aging bladder dysfunction. Ther Adv Urol 2017;9:11-27.

15. Azadzoi KM, Yalla SV, Siroky MB. Oxidative Stress and Neurodegeneration in the Ischemic Overactive Bladder. J Urol 2007;178:710-5.

16. Azadzoi KM, Radisavljevic ZM, Siroky MB. Effects of ischemia on tachykinin-containing nerves and neurokinin receptors in the rabbit bladder. Urology 2008;71:979-83.

17. Azadzoi KM, Chen BG, Radisavljevic ZM, et al. Molecular Reactions and Ultrastructural Damage in the Chronically Ischemic Bladder. J Urol 2011;186:2115-22.

18. Nomiya M, Burmeister DM, Sawada N, et al. Prophylactic Effect of Tadalafil on Bladder Function in a Rat Model of Chronic Bladder Ischemia. J Urol 2013;189:754-61.

19. Nomiya M, Burmeister DM, Sawada N, et al. Effect of melatonin on chronic bladder-ischaemiaassociated changes in rat bladder function. BJU Int 2013;112:E221-30.

20. Yang JH, Siroky MB, Yalla SV, et al. Mitochondrial stress and activation of PI3K and Akt survival pathway in bladder 
ischemia. Res Rep Urol 2017;9:93-100.

21. Chen MW, Buttyan R, Levin RM. Genetic and cellular response to unilateral ischemia of the rabbit urinary bladder. J Urol 1996;155:732-7.

22. Gill HS, Monson FC, Wein AJ, et al. The Effects of Short-term In-vivo Ischemia on the Contractile Function of the Rabbit Urinary Bladder. J Urol 1988;139:1350-4.

23. Lin ATL, Wein AJ, Gill HS, et al. Functional effect of chronic ischemia on the rabbit urinary bladder. Neurourol Urodyn 1988;7:1-12.

24. Vince R, Tracey A, Deebel NA, et al. Effects of vesical and perfusion pressure on perfusate flow, and flow on vesical pressure, in the isolated perfused working pig bladder reveal a potential mechanism for the regulation of detrusor compliance. Neurourol Urodyn 2018;37:642-9.

25. Anele UA, Ratz PH, Colhoun AF, et al. Potential vascular mechanisms in an ex vivo functional pig bladder model. Neurourol Urodyn 2018;37:2425-33.

26. Tracey AT, Anele UA, Vince RA, et al. Bladder attack:

Cite this article as: Cullingsworth ZE, Nandanan N, Swavely NR, Frolov K, Vince R, Zee R, Cisu T, Klausner AP, Speich JE. A preliminary study of bilateral color mapping of pig bladder vasculature demonstrates potential for acute hemi-ischemic events. Transl Androl Urol 2021;10(6):2410-2417. doi: 10.21037/ tau-20-1467 transient bladder ischemia leads to a reversible decrease in detrusor compliance. Transl Androl Urol 2019;8:703-11.

27. Balthazar A, Cullingsworth ZE, Nandanan N, et al. An external compress-release protocol induces dynamic elasticity in the porcine bladder: A novel technique for the treatment of overactive bladder? Neurourol Urodyn 2019;38:1222-8.

28. Swavely NR, Cullingsworth ZE, Nandanan N, et al. Phases of decompensation during acute ischemia demonstrated in an ex vivo porcine bladder model. Transl Androl Urol 2020;9:2138-45.

29. Gillispie T, Iii S, Coburn M. Urologic Surgery. In: Townsend C, Beauchamp RD, Evers BM, et al. editors. Sabiston Textbook of Surgery 20th ed. Elsevier Inc., 2019.

30. Juan YS, Li S, Levin RM, et al. The effect of ischemia/ reperfusion on rabbit bladder--role of Rho-kinase and smooth muscle regulatory proteins. Urology 2009;73:1126-30. 